\title{
Energy Efficient Data Aggregation in Wireless Sensor Networks using Mobile Sink Node
}

\author{
Meenakshi Bansal, Ashok Kumar Bathla
}

\begin{abstract}
The wireless sensor networks consist of numerous small nodes which are also called as energy resource-constrained sensor nodes. The communication of these nodes can be done in a various way. There is also the processing of signal tasks which is done through the various computational resources provided by the networks. The energy of the sensor nodes gets consumed when transmit the data or receive data from the network. To reduce energy consumption of the network various techniques has been proposed which are known as clustering techniques. In the proposed work the mobile sink is deployed in the network which reduces overhead in the network. Experimental results shows that the proposed work outperforms the existing one in terms of reduced energy consumption of the network, increased throughput of the network, reduced delay in the network.
\end{abstract}

Keywords: Wireless Sensor Network, Energy Consumption, Throughput, Packet delay, Mobile Sink.

\section{INTRODUCTION}

Wireless sensor network is progressing with latest upcoming technologies and development in the system. These types of networks are used commonly in every field because of their typical characteristics that are very helpful to do the every task in every system. The structure of the wireless sensor networks system is very difficult and complex that is designed according to the requirement of the system in the field. The energy issue is mainly considerable in these types of networks because the whole system requires the efficient energy to perform the particular task. The system becomes very large due to the increasing number of nodes that are used to transfer or receive the data from other resources in the system, which is used to communicate with each other within the system [1]. There are many other tiny nodes are used that are useful to the energy resource. The communication within the whole system is processed by the wireless system that provides the signal to the nodes according to the requirement of the data communication.

\subsection{Types of Wireless Sensor Networks (WSN)}

Classifications of the WSNs on the basis of located networks are as the following:-

Terrestrial WSN: In the ad hoc network the different nodes are collected at a place that has the lower power. The high power resources provide the energy to these sensor nodes.

Revised Manuscript Received on December 30, 2019.

* Correspondence Author

Meenakshi Bansal*, Assistant Professor, Yadavindra College of Engineering, Guru Kashi Campus, Talwandi Sabo.

Ashok Kumar Bathla, Assistant Professor at Yadawindra College of Engineering, Guru Kashi Campus, Talwandi Sabo

(C) The Authors. Published by Blue Eyes Intelligence Engineering and Sciences Publication (BEIESP). This is an open access article under the CC BY-NC-ND license (http://creativecommons.org/licenses/by-nc-nd/4.0/)
The communication is mainly done between the sensor nodes and the base station to interchange the information.

Underground WSN: This network is constructed for the underground systems. These networks provide the information and told about the conditions from the underground areas. There is a sink constructed underground to communicate with the base station and deliver the information for the next step to do.

Underwater WSN: The underwater wireless sensor networks are made to know about the underwater conditions. There are some types of underwater vehicles which are helpful to construct the wireless sensor network in the underwater. These vehicles transfer the information to the base station. This system is very costly as compared to the other conditions of networks.

Multi-media WSN: The wireless sensor network is also used to transfer the multimedia data like as audios and videos from one place to another place by using the sensor nodes. There is a special area in which the multimedia is transferred in the system.

Mobile WSN: This network is very popular these days. The mobile network is the big need of everyone in the developing world. It is mainly used for communicating with other people and also used for sensing the external environment like the temperature of a particular area [2].

\subsection{Sensor Network Architecture}

The following components are used to operate the functions in the wireless sensor network.

a. Gateway: There is an interconnection between the user and the wireless sensor nodes that is called the gateway. The gateway is mainly used for transferring the information between two connected nodes. The same procedure can be applied for the reverse position.

b. Base Station: The internet provides the interconnection between the user and the base station. The base station is that who fulfil the requirement of sending and receiving the data from every part of the network [3]

c. Sensor node: These are the tiny parts of the network that provide the main connection between the sender and the receiver. The whole system depends on these nodes because these are used for transferring the data from one to another stage.

d. Relay node: The router is another name of the relay node. It is connected to full-function devices (FFD). This can be used for the back-ups of the data which is transferred within the network. It increases the coverage area and also tackles the problems that are produced in the system during the transaction of information.

\subsection{Clustering}

In the wireless sensor network there is several number of sensor nodes are attached to each other to make a wide network according to the requirement of the area.

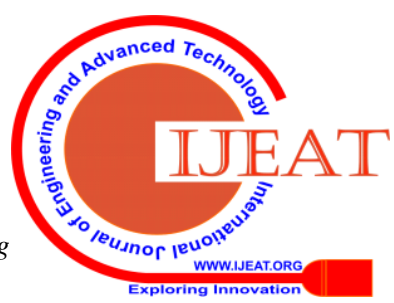




\section{Energy Efficient Data Aggregation in Wireless Sensor Networks using Mobile Sink Node}

To make this type of network the dimensions of the area is not calculated. The network is basically formed according to the requirement and the functions which are performed by that network. The timing of the network is not limited because it is needed for a long time like as in the army area and in the wild life area. Once it is installed then it works for a long time of intervals because it becomes more costly to reconstruct the wireless sensor network. The large amount of area is required to implement the wireless sensor network because the number of sensor nodes need to install in the field where we need to apply the network. The power of battery is needed to provide each and every sensor node because it works by using the battery power. The security is also needed to these types of networks. It becomes very difficult to provide the battery power to every node within the networks [4]. Along these lines, shifted methods are creating to supply the vitality utilization of hubs. It is conjointly the different conventions are required for this strategy. The inclusion of conventions and their decision are imperative elements. All these influence the system framework by and large in different behavior.

Cluster Head: Firstly the clusters are developed and then the centroid point is calculated in the network of each cluster. This method mainly works on the position of the node and then according to the location of that node is selected as a cluster head. The new participated nodes also can be selected as the cluster head after calculating the centroid point of those nodes. The distance gap between the cluster nodes has to minimum to calculate the location of the centroid. In some conditions the threshold value varies and become low then the cluster head is chosen again on the basis of highest energy and minimum distance [5].

Mobile Sink Nodes: The number of nodes is increasing day by day in the network that requires more energy to implement and handle all those nodes. These nodes have to consume less amount of energy and give more performance as compared to the old one. There are number of networks that are disabling to handle the small range of nodes that are not in the scale [6]. They are set to be at the intervals of time to get the best results. This methodology contains the following factors:

- To design the large scale wireless sensor networks the multiple mobile sinks are involved in the system.

- In the larger wireless sensor networks the graphical representation is used to separation the large networks.

- The number of sinks is added in the wireless sensor networks to minimize the energy consumption.

\section{RELATED WORK}

Lotfinezhad et al. [7] the work was done in this paper on the basis of energy consumption, latency and robustness. The performance of the network was mainly considerable by making the clustering and data correlation jointly with each other. The simple Medium Access Control (MAC) was used by cluster heads in this paper to overcome the weakness of data sink. The main method in this paper used CDC-DSA which is used to make the clustering as a randomly. In conclusion, the designing of the clusters decreased the energy consumption and the rate of latency as compared to other techniques. Heo et al. [8] the proposed technique in this was on the behalf of path selection. That path is chosen to deliver the data. Which consumes less energy and able to deliver the packet in real time. The proposed method was EARQ, which was made to use in the industrial phase to save the time and remove the workload. This method chooses the path to that node. Which has lower energy and it enhances the energy level and speed of the data transmission.

Chagas et al. [9] the work was done in this paper was on the basis of energy consumption during the data storage and to correct the problems that occurs during the transmission of data only in the online system. The technique was need in this paper was distribution of the data that means divided the data in small amounts at the storage time. In this paper the work done on the same device directly that is required to gather the information because it consumes less energy at the real time. The data is sent in the small amounts of time intervals.

Han et al. [10] this paper defines the drawbacks of the wireless sensor network. This is deployed in heterogeneous system. There is number of relay nodes are attached for the fault tolerance. The author describes the method of homogeneous wireless sensor network in which the relay nodes are attached to transfer the data in the network. Firstly, it works on the basis of radius of the relay node. The radius of every relay node is different from others and the nodes are selected in which path the numbers of relay nodes are minimum as the vertex disjoint paths. There are two structures of paths that can be selected as the transmission of information first is two path in which the data can be sent in both in which the and second is one way path in, which the information can be shared only in a single direction. This paper defines the number of problems are related to fault tolerance.

Lee et al. [11] in the communication system the number of nodes, sink and users are involve making the communication better and faster. There are mainly two types of users the first one is mobiles users that move one place to another place to communication with others and the second one is traditional remote users that are fixed on the one location to get or transfer the information. The author explained the both techniques in this paper and proposed a method that works in a legacy network in which the multiple static sinks are attached to make the communication better in less time and consumes less energy and provide better throughput in the wireless sensor network. Logambal et al. [12] in this paper, the techniques were discussed that in related to the health problems, agriculture, energy, privacy, security, transportation and so on. These are the main parts of the challenges that are named in this paper because the whole system cannot be defined in a single paper. There are many health problems which cannot be detected directly by the doctors. In the condition there are some sensors, which are used to detect the problem internally or externally in the human body.

\section{METHODOLOGY}

In this work the main aim is to increase the energy efficiency of the network by using the multiple sink modes. The sinks take a movement from their existing location to make the communication easy and energy effective. The proposed method is combined with the LEACH protocol. The movement of the sinks depends upon the bee colony algorithm and the strength of the signal. 
By using the combination of LEACH protocol and multiple mobile sink modes, the performance of the network increased and energy consumption decreased. The following steps of flowchart used in the proposed method.

Step-1: Firstly applied the limited number of nodes in the wireless sensor network and distributed to provide the fixed form clusters using location based clustering.

Step-2: In the next step LEACH protocol is checked the maximum energy and minimum distance is used to other nodes is fixed as the cluster head. Another node in the cluster aggregates their data to the cluster head.

Step-3: The locations is changed according to the signal strength and take a move on the basis of starting population and aggregate the data from where it gets maximum data.

Step-4: The step number 3 is repeated again and again until the needed data aggregated to the base station.

Figure 1 describes the process of proposed work. At the first the limited number of nodes is implemented in the wireless sensor network to form the clusters on the basis of location. To make the cluster head the LEACH protocol is used by calculating the maximum energy and minimum distance. The other nodes aggregate data with the cluster head. The location of the cluster head is changed to aggregate the maximum data with the cluster nodes, on the basis of the signal strength. This process continuous working on till the data is aggregated with the base station.

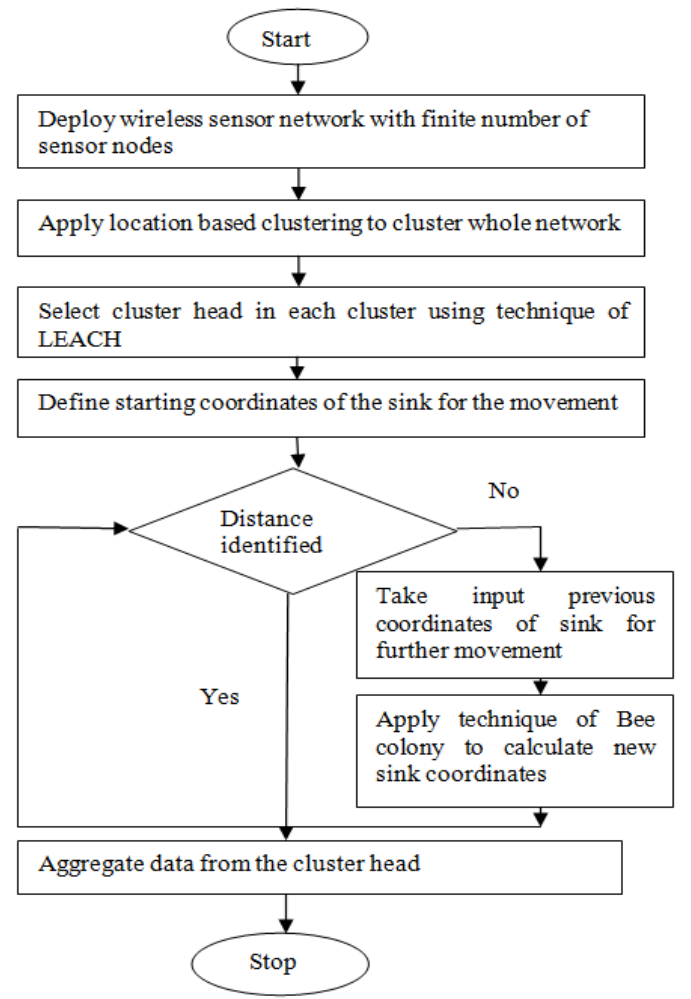

\section{RESULTS AND DISCUSSION}

The results of the proposed method is presented and discussed in this section. The whole scenario is implemented on Network Simulator2 (NS2). It is a simulation and discrete event simulator.

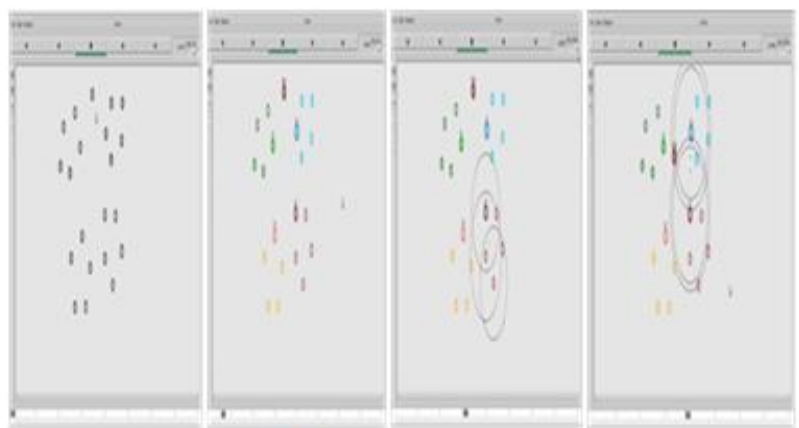

(a)

(b)

(c)

(d)

Fig. 2. (a) Deployment of network (b) Clustering of network (c) Data aggregation (d) Data aggregation between clusters

Firstly applied the limited number of nodes in the wireless sensor network as shown in figure 2 (a) and distributed to provide the fixed form clusters using location based clustering.

As shown in figure 2 (b), the whole network is checked by the LEACH protocol in which the maximum energy and minimum distance is used to other nodes to fix as the cluster head.

Another node is used in the cluster aggregates their data to the cluster head.As shown in figure 2 (c), another node in the cluster aggregates their data to the cluster head. The location is changed according to the signal strength and takes a move on the basis of starting population and aggregate the data from where it gets maximum data. The data from the sensor nodes is aggregated to the cluster head. As shown in figure 2 (d), the cluster heads aggregate their data with each other that is collected from the sensor nodes of the clusters.

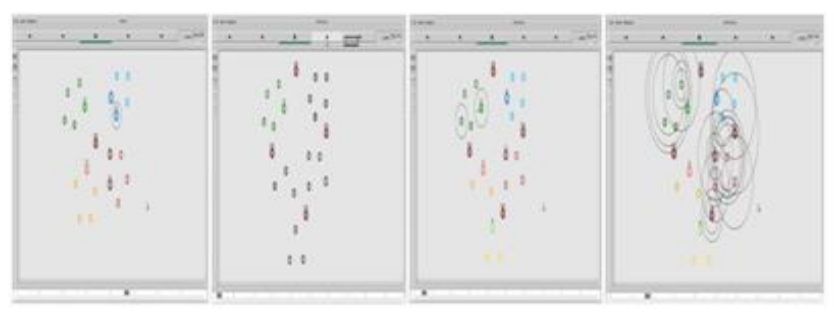

(a)

(b)

(c)

(d)

Fig. 3. (a) Movement of base station (b) Network deployment (c) Data aggregation between cluster head and nodes (d) Data aggregation

As shown in figure 3 (a), the base station move according to the data requirement and the energy efficiency.

As shown in figure 3 (b), the multiple sinks are generated to aggregate the data within the clusters that is required to the sensor nodes. As shown in figure 3 (c), the data is aggregated between the clusters heads and the sensor nodes to share the data with other clusters. As shown in figure 3 (d), the data is aggregated between the sinks and the cluster heads by the communication with each other.

Table 1Comparison between Proposed method and Existing technique on the basis of Energy consumption

\begin{tabular}{|l|l|l|l|}
\hline Parameter & $\begin{array}{l}\text { Time } \\
\text { (sec) }\end{array}$ & $\begin{array}{l}\text { Existing } \\
\text { technique }\end{array}$ & $\begin{array}{l}\text { Proposed } \\
\text { technique }\end{array}$ \\
\hline $\begin{array}{l}\text { Energy } \\
\text { consumption }\end{array}$ & $10 \mathrm{sec}$ & 75 joules & 30 joules \\
\hline
\end{tabular}




\begin{tabular}{|l|l|l|l|}
\hline Packet loss & $10 \mathrm{sec}$ & 38 packets & 15 packets \\
\hline Throughput & $10 \mathrm{sec}$ & 14 packets & 18 packets \\
\hline
\end{tabular}

Table 1 depicts that energy consumption in existing technique is very much less as compare to the existing technique. Second parameter i.e packet loss is also reduced in the proposed technique and finally the through put is improved.

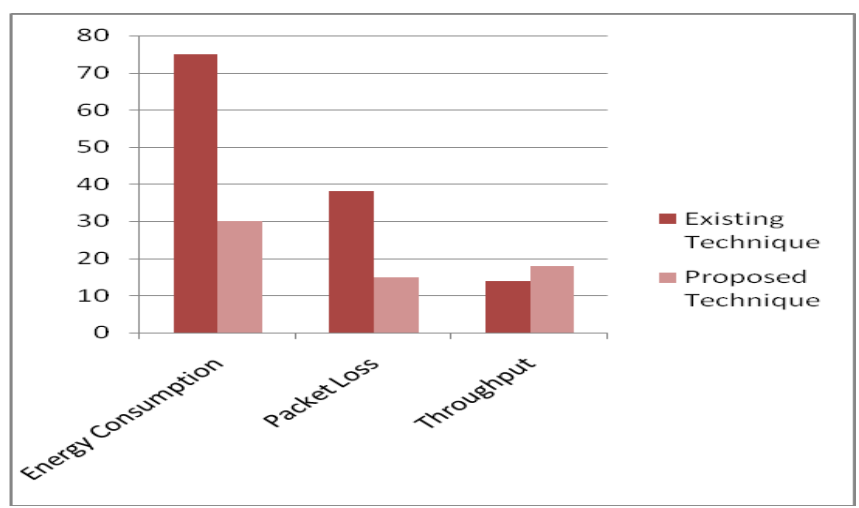

Fig. 4. Graph for comparison of proposed method with existing one.

Graph in Figure 4 , depicts the representation of existing as well as proposed technique. The dark bar illustrates the functions performed by existing technique and the light bar defines the functions performed by proposed technique. The performance of proposed technique is better than existing technique in terms of energy consumption, packet loss and throughput. The graph illustrates that energy consumption of the proposed technique is less than the half of the energy consumed by the existing technique. The packet loss is also same as the criteria of energy consumption that means packet loss is always more in existing method as compared to the proposed technique. The throughput of the proposed method is much better than the existing technique.

\section{CONCLUSION}

In this paper the various wireless sensor network techniques are studied. The proposed method is combined with LEACH protocol. The movement of the sinks depends upon the bee colony algorithm and the strength of the signal. The proposed method is used to increase the energy efficiency of the network by using the multiple sink modes. The sinks take a movement from their existing location to make the communication easy and energy effective. By using the combination of LEACH protocol and multiple mobile sink modes, the performance of the network increased and energy consumption and packet loss decreased. The collection and transformation of data became easy. The lifetime of the wireless sensor network increased for a long time.

\section{REFERENCES}

1. Willig, A., 2006. Wireless sensor networks: concept, challenges and approaches. e \& i Elektrotechnik und Informationstechnik, 123(6), pp.224-231.

2. Rawat, P., Singh, K.D., Chaouchi, H. and Bonnin, J.M., 2014. Wireless sensor networks: a survey on recent developments and potential synergies. The Journal of supercomputing, 68(1), pp.1-48.

3. Cecílio, J. and Furtado, P., 2014. Wireless sensor networks: concepts an components. In Wireless Sensors in Heterogeneous Networked Systems (pp. 5-25). Springer, Cham.

4. Chuang, P., Yang, S., and Lin, C., 2009. Energy-Efficient Clustering in Wireless Sensor Networks, Springer Algorithms and architecture for parallel processing of the series Lecture notes in computer science, volume 5574, pp 112-120.

5. Jerusha, S., Kulothungan, K. and Kannan, A., 2012. Location aware cluster based routing in wireless sensor networks. International Journal of Computer and Communication Technology, 3(5), pp.1-6.

6. Slama, I., Jouaber, B. and Zeghlache, D., 2008, July. Multiple mobile sinks deployment for energy efficiency in large scale wireless sensor networks. International Conference on E-Business and Telecommunications (pp. 412-427). Springer, Berlin, Heidelberg.

7. Lotfinezhad, M., Liang, B. and Sousa, E.S., 2008. Adaptive cluster-based data collection in sensor networks with direct sink access. IEEE Transactions on Mobile computing, 7(7), pp.884-897.

8. Heo, J., Hong, J. and Cho, Y., 2009. EARQ: Energy aware routing for real-time and reliable communication in wireless industrial sensor networks. IEEE Transactions on Industrial Informatics, 5(1), pp.3-11.

9. Chagas, L.D., Lima, E.P. and Neto, P.F.R., 2010, March. Real-time databases techniques in wireless sensor networks. In Networking and Services (ICNS), 2010 Sixth International Conference on (pp. 182-187). IEEE.

10.Han, X., Cao, X., Lloyd, E.L. and Shen, C.C., 2010. Fault-tolerant relay node placement in heterogeneous wireless sensor networks. IEEE Transactions on Mobile Computing, 9(5), pp.643-656.

11. Lee, E., Park, S., Yu, F. and Kim, S.H., 2010. Communication model and protocol based on multiple static sinks for supporting mobile users in wireless sensor networks. IEEE Transactions on Consumer Electronics, 56(3).

12. Logambal, M., and Thiagarasu, V., 2017. A survey on wireless sensor network in human healthcare monitoring system, International Journal of Innovative Research in Computer and Communication Engineering, 5(4), pp.7635-7641.

\section{AUTHORS PROFILE}

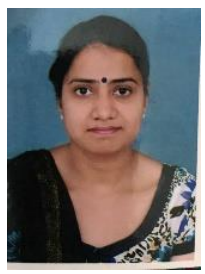

Meenakshi Bansal is currently working as Assistan Professor at Yadavindra College of Engineering, Guru Kashi Campus, Talwandi Sabo. She is doctorate in the area of Datamining and Network Security and has completed her Ph.D from I K Gujral, Punjab Technical University, Kapurthala in 2019. She has completed her Master's degree in computer Science and Engineering from Punjab Agriculture University, Ludhiana in 2006 and supervised 42 students of M.Tech in different area of Computer Engineering and Graduation from I K Gujral, Punjab Technical University, Kapurthala in 2004. She has nearly 50 research papers published to her credit in reputed journals and conferences.

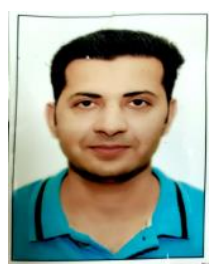

Ashok Kumar Bathla is currently working as Assistant Professor at Yadawindra College of Engineering, Guru Kashi Campus, Talwandi Sabo. He was born on $31^{\text {st }}$ August, 1981 at Malout, Punjab, India. He is Pursuing doctorate (Thesis Submitted) in the area of Natural Language Processing viz Optical Character Recognition. He is doing his Ph.D from I K Gujral, Punjab Technical University, Kapurthala. He has completed his Master's degree in computer Science and Engineering from Punjabi University, Patiala in 2008 and Graduation in the same branch from I K Gujral, Punjab Technical University, Kapurthala in 2004. He has supervised more than 25 students of M.Tech in different area of Computer Engineering and he has nearly 50 research papers published to his credit in reputed journals and conferences. For the last 14 years, he is actively involved in writing, teaching and research in the diverse areas of Computer Technology. 